\title{
Investigations on Microbial Flora Variation in Water Generated at Various Sugar Production Stages
}

\author{
Seema Paroha* and Shikha Singh \\ National Sugar Institute, Kanpur, India \\ *Corresponding author
}

\begin{abstract}
A B S T R A C T
\section{Keywords}

Bacteria, fungi, sugar industries, effluent

\section{Article Info}

Accepted:

10 April 2020

Available Online:

10 May 2020

The water samples of different sugar producing stages from eight selected sugar factories were collected to explore the possibility of its utilization as fresh water. Samples were collected from Fresh / Raw water; $1^{\text {st }}$ body condensate; $2^{\text {nd }}$ body condensate; $3^{\text {rd }}$ body condensate; $4^{\text {th }}$ body condensate; Pan condensate; UGR Cold; UGR Hot; ETP Inlet and ETP Outlet. On an average the macroscopic characters exhibited off white colour, round shape, flat elevation and rough/slimy appearance in all 8 sugar factories whereas microscopic characters of these water samples showed rod /cocci shape and both gram positive and grams negative bacteria in all eight selected sugar factories except vapour condensates exhibited negligible growth of the same. This indicates that vapour condensates are most promising and can be converted into fresh water after required processing. Various Bacterial strains were identified viz E.coli, B.subtilis, P.putida, Salmonella spp, S.aureus and B.cereus etc. It was observed that fungal colony was only seen in water sample of fresh water and in ETP inlet and outlet. Maximum numbers of fungal species identified were Alternaria gaisen, Aspergillus flavus, Aspergillus candidus, Aspergillus niger, Aspergillus nidulans, Peniciilium pinophillum and Trichoderma viride, this database created a novel record of the fungal diversity associated with sugarcane industrial effluent
\end{abstract}

\section{Introduction}

The most important crop from which sugar can be produced in commercial quantity are sugarcane. India is a largest sugar producing country. Sugar industry has been classified in solid, liquid, and gas Pawar et al., (1998) under seventeenth categories of most polluting industries (Red industry) by the central pollution control board (CPCB) in India CREP-CPCB, (2003). Sugar mills account in the industries which discharge huge amount of effluent per day without any or partially treatment during the crushing season Vinish kathuria, (2014).

Government has certain norms due to control water because it is the main raw material for production process, in each and every stage of manufacturing has lots of complicity on utilizing water in proper manner. The wastewater generated by the sugarcane industries are characterized by their high levels of BOD, COD, $\mathrm{pH}$, TSS and TDS. 
Sugar cane industry generates $0.2-1.8$ $\mathrm{m} 3 /$ tonne waste water with COD 1800 to $3200 \quad \mathrm{mg} / \mathrm{L}, \quad$ BOD $720-1500 \quad \mathrm{mg} / \mathrm{L}$. Rajeshwarai \& Balakrishnan (2000) which are causes for developing water pollution when left untreated. The reason for because sugar industry effluent have high organic matter content such as sugar oils, fats, grease and proteins which have bad impact on the quality of ground water Brown \& Skougstad (1970).

Boiler house mainly contributes to the production of air pollution and have little share in water pollution Bevan (1971)This leaves a foul smelling (Hydrogen sulphide) gas which in turn can precipitate iron and any dissolved salts, turning the water black and highly toxic for aquatic life. The dissolved oxygen should be normally at least $5 \mathrm{mg} /$ litre for the survival of fishes and other aquatic life Avasan \& Rao (2009).

Discharge of water with high TDS level would have adverse impact on aquatic life, render the receiving water unfit for drinking, reduced crop yield if used irrigation, and exacerbate corrosion in water system and pipe. Sugar factory effluent produces obnoxious odour and unpleasant color when released into the environment without proper treatment. Farmers have been using these effluents for irrigation, found that the growth, yield and soil health were reduced. Along with the effects of various industrial effluents on seed germination, growth and yield of crop plants have captivated the attention of many workers Rahman et al., (2002, Street et al., (2007).

As we know sugar is organic molecule and process of sugar itself contain organic matter which when come in various sections escape in form of vapours or dissolved form in various streams. Thus wastewater of sugar industry contains high levels of organic matter, which serves as food for Bacteria. This results in the increase of these microorganisms in the water. The increased number of bacteria then use up all the dissolved oxygen in the water. On complete exhaustion of oxygen the bacteria begin to break down the chemicals in the water stream to get them oxygen.

The life in effluent is highly diverse and consists of interacting population of microorganisms and effluent fauna, and their activities affect physical, chemical, and biological characteristics of effluent. Microbes are the only entities in the biosphere with an exceptional ability to exploit various organic and inorganic compounds for their growth and transform them to chemical products no longer hazardous to human health and the environment. Some potential fungal and bacterial strains were isolated from sugarcane industrial effluent. Pant and Adholeya (2007b). The aim of this study is to find out pattern of distribution of different microorganisms found in water sample of Sugar Industry collected from different sugar production stages were studied.

\section{Materials and Methods}

\section{Collection of water sample}

The 8 sugar-mill water samples were collected. Three from the Uttar Pradesh, three from Maharashtra and two from Tamil nadu in sterilized bottles tightly capped. Water samples collected from different sugar production stages were screened for the isolation of potential bacterial and fungal strains. Following samples were collected from the sugar mill-

Fresh / Raw water. 1 st body condensate. 2nd body condensate. 3rd body condensate. 
4th body condensate

Pan condensate.

UGR Cold.

UGR Hot.

ETP Inlet.

ETP Outlet.

\section{Isolation of bacteria}

Samples after being serially diluted in sterile distilled water were plated on nutrient agar plates and then incubated for $48 \mathrm{hrs}$ at $30^{\circ} \mathrm{C}$. Discrete bacterial colonies that grew on agar plates were initially grouped on the basis of gram staining and different morphological characteristics such as pigmentation motility and colony forms. Bacterial isolates were then picked, sub-cultured and subjected to further biochemical tests for identification according to Bergey's manual of Determinative Bacteriology.

\section{Physiological and biochemical tests}

The physiological and biochemical tests were conducted following the methods of Somasegaran and Hoben (1985) and Josey et al., (1979) respectively, as described by Cappuccino and Sherman (1999) to identify the bacteria. and also confirmed with the help of PIB computer kit. Bryant, (1989].

\section{Isolation of fungi}

$1 \mathrm{ml}$ of the water sample was taken in a $250 \mathrm{ml}$ conical flask containing $90 \mathrm{ml}$ sterile distilled water. The flask was shaken on an electric shaker to get a homogenous suspension and transferring serially $10 \mathrm{ml}$ of the water suspension to $90 \mathrm{ml}$ of sterile distilled water made different dilution viz., $10^{-1,} 10^{-2}$ and $10^{-3}$. $1 \mathrm{ml} 10^{-3}$ dilution was and plated in petridishes containing Potato Dextrose Agar medium (PDA). The $\mathrm{pH}$ of the medium was adjusted to 5.6. Streptomycin sulphate (100 $\mathrm{ml}$ ) was added to the medium to prevent the bacterial growth. The plates were incubated at $25+2^{0} \mathrm{C}$ for five days and fungi appearing on the medium were mounted over a clean slide, stained with lacto phenol cotton blue and observed under the microscope. The fungi were identified by using standard manuals, such as Manual of soil fungi Gillman, (1957). More Dematiaceous Hyphomycetes Ellis, (1976) and Hyphomycetes Subramanian, (1971).

\section{Findings}

For the present study, the microbial diversity of water and waste water was carried out. The abundant variation in the types of microorganisms and their distinctive morphological and anatomical features visible to the naked eye enable us to identify the different genera and species and, therefore we can easily assess the extent of their diversity.

Hence, the present study was undertaken to know the bacterial and fungal in different water and waste water samples. For the present investigation samples were collected from 8 sugar factory, three from the Uttar Pradesh, three from Maharashtra and two from Tamilnadu in sterilized bottles tightly capped. Following samples were collected from the sugar mill-Fresh / Raw water; 1st body condensate; 2 nd body condensate; 3rd body condensate; 4th body condensate; Pan condensate; UGR Cold; UGR Hot; ETP Inlet and ETP Outlet;. The bacterial and fungal species were isolated and identified from water samples were recorded.

\section{Visual observation}

Samples after being serially diluted in sterile distilled water were plated on nutrient agar plates and then incubated for $48 \mathrm{hrs}$ at $30^{\circ} \mathrm{C}$.The results of investigation revealed that the water samples from Pan Condensate, ETP Inlet, ETP outlet, Hot UGR, Cold UGR, 
including fresh water samples from all eight selected factories contained microscopic and macroscopic elements, whereas samples of all the vapour condensate exhibited negligible growth of the same. This indicates that vapour condensates are most promising and can be converted into fresh water after required processing. On an average the macroscopic characters of fresh water samples, Pan Condensate water, Hot UGR water Cold UGR water ETP Inlet water and ETP outlet water exhibited off white colour, round shape flat elevation and rough/slimy appearance in all 8 Sugar Factories whereas microscopic characters of these water samples showed rod /cocci shape and both gram positive and grams negative bacteria in all eight selected sugar factories (Table 1-8).

\section{Bacterial flora in the collected samples}

Bacteria were isolated from the water samples by serial dilution techniques. Identification of Bacterial Strains was done based on Biochemical Test evaluation. (Table No.916).The Biochemical Test investigation of obtained bacterial population revealed that the bacterial strain viz E.coli, B.subtilis, P.putida, Salmonella spp, S.aureus and B.cereus etc were present in the samples of fresh water samples, Pan Condensate water, Hot UGR water, Cold UGR water, ETP Inlet water and ETP outlet water in general whereas vapour condensates samples exhibited negligible growth of the same. This indicates that vapour condensates are most promising and can be converted into fresh water after required processing. Ramlake and Bhattacharjee (1992) suggested the polluted habitats found mostly Pseudomonas because it is having ability to degrade various pollutants from water samples. Most studies on the metabolism of organic contaminants have been performed with bacteria especially in the context of bioremediation Glazer, (1997). Bacteria generally are easier to culture and they grow more quickly than fungi. They are more amenable to molecular genetic manipulations. They are able to metabolize chlorinated and other organic contaminants such as oil and mineralize chemicals using them as carbon or energy source Glazer, (1997). Dahiya et al., (2001a) isolated Pseudomonas fluorescens from reactor liquid and found that these bacterial strains are capable of decolourizing melanoidin wastewater up to $76 \%$ under non-sterile condition and upto $90 \%$ in sterile condition. Decolorization of industrial effluents has been achieved by degradation using bacterial and fungal isolates Suhuttaya, (2009).

According to the result of microbial analysis revealed that the water samples from the Uttar Pradesh factories (I, II, III) Maharashtra sugar mills (IV, V, VI) , Tamil Nadu (VII, VIII) contained bacteria from the family Enterobactericea which are facultative anaerobes, coliforms. They produce mixed acid fermentation. As fresh water contains millions of micro flora these microbes are found in abundance. But not all these microbes are pathogenic accept Salmonella spp. and Klebsiella spp. which may cause some water borne diseases.

\section{Fungal flora in the collected samples}

The Fungal evaluation and identification was done in the selected water samples. It was observed that fungal colony was only seen in water sample of fresh water and in ETP inlet and outlet. For identification of fungus culture was mounted on clean slides and stained with lacto phenol cotton blue. The slides were observed under the microscope. The fungus strains were identified based on colony characteristics and staining methods. The mycofloristic composition of effluent sample of different industries varied significantly. Maximum numbers of fungal species identified in all Effluent outlet water samples 
were Alternaria gaisen, Aspergillus flavus, Aspergillus candidus, Aspergillus niger, Aspergillus nidulans, Peniciilium pinophillum and Trichoderma viride. The genera Aspergillus was frequently found in sugar industrial effluent of different sugar factories. Table No. 17. In the course of this study the total 8 fungus species belonging to 7 genera which were capable to degrade the organic matter was observed.

Some potential fungal strains such as Penicillium pinophilum, Alternaria gaisen, Aspergillus flavus, Fusarium monolifome, A.niger were also isolated and reported from sugarcane industrial effluent (Pant and Adholeya 2007) A total number of 15 species belonging to 9 genera of fungi were isolated during our investigation in various sugarcane industries of Madhya Pradesh by Awasthi et al., (2011). Diverse fungal cultures have been investigated recently for bioremediation process Aust, (1990) and Bumpus and Aust (1993). By virtue of their aggressive growth, greater biomass production and extensive hyphal reach in the environment, fungi have been seen to perform better than bacteria. The high surface -to - cell ratio of filamentous fungi makes them better degraders under certain niches Ashoka et al., (2002). The fungus have capability to purify the effluent by consumption of organic substances, thus, reducing its COD and BOD, and at the same time to obtain some valuable product, such as fungal biomass for protein rich animal feed or some specific fungal metabolite.

One of the most studied fungus having ability to degrade and decolourize distillery effluent is Aspergillus sps. such as Aspergillus fumigatus G26, A. niger, A. niveus, $A$. fumigates $\mathrm{Ub}^{2} 60$ brought about an average of $69.75 \%$ de-colourization along with $70-90 \%$ COD reduction Ohmomo et al., (1987); Miranda et al., (1996); Jimnez et al., (2003), Radhika Agarwal et al., (2010). It is evident from the available data that microbial composition of effluent sample of industry varied significantly. Maximum numbers of fungal species were recorded compare to bacteria

Table.1 Macroscopic and Microscopic visual characters of water samples from different sugar production stages of Uttar Pradesh Sugar Factory -I

\begin{tabular}{|c|c|c|c|c|c|c|c|}
\hline \multirow[t]{2}{*}{ S.No } & \multirow[t]{2}{*}{ Sample } & \multicolumn{4}{|c|}{ Macroscopic Characters } & \multicolumn{2}{|c|}{ Microscopic } \\
\hline & & Colour & Shape & Elevation & Appearance & Shape & Gram stain \\
\hline 1. & Fresh water & Off White & Round & Flat & Rough & Rods & G-ve \\
\hline 2. & $1^{\text {st }}$ body condensate & \multicolumn{6}{|c|}{ Negligible Growth } \\
\hline 3. & $2^{\text {nd }}$ body condensate & \multicolumn{6}{|c|}{ Negligible Growth } \\
\hline 4. & $3^{\text {rd }}$ body condensate & \multicolumn{6}{|c|}{ Negligible Growth } \\
\hline 5. & $4^{\text {th }}$ body condensate & \multicolumn{6}{|c|}{ Negligible Growth } \\
\hline 6. & Pan Condensate & Off White & Round & Flat & Rough & Rods & G-ve \\
\hline 7. & Hot UGR & Off White & Round & Flat & Slimy & Rods & G+ve \\
\hline 8. & Cold UGR & Off White & Round & Flat & Rough & Rods & G-ve \\
\hline 9 & ETP Inlet & Off White & Round & Flat & Rough & Rods & G-ve \\
\hline 10 & ETP outlet & Off White & Round & Flat & Slimy & Cocci & G+ve \\
\hline
\end{tabular}


Table.2 Macroscopic and Microscopic visual characters of water samples from different sugar production stages of Uttar Pradesh Sugar Factory -II

\begin{tabular}{|c|c|c|c|c|c|c|c|}
\hline \multirow[t]{2}{*}{ S.No } & \multirow[t]{2}{*}{ Sample } & \multicolumn{4}{|c|}{ Macroscopic Characters } & \multicolumn{2}{|l|}{ Microscopic } \\
\hline & & Colour & Shape & Elevation & Appearance & Shape & Gram stain \\
\hline 1. & Raw water & Off White & Round & Flat & Rough & Rods & G-ve \\
\hline 2. & $1^{\text {st }}$ body condensate & \multicolumn{6}{|c|}{ Negligible Growth } \\
\hline 3. & $2^{\text {nd }}$ body condensate & \multicolumn{6}{|c|}{ Negligible Growth } \\
\hline 4. & $3^{\text {rd }}$ body condensate & \multicolumn{6}{|c|}{ Negligible Growth } \\
\hline 5. & $4^{\text {th }}$ body condensate & \multicolumn{6}{|c|}{ Negligible Growth } \\
\hline 6. & Pan condensate & Off White & Round & Flat & Slimy & Rods & G-ve \\
\hline 7. & Hot UGR & Off White & Lawn & Flat & Slimy & Cocci & G-ve \\
\hline 8. & Cold UGR & Off White & Round & Flat & Slimy & Cocci in chains & $\mathrm{G}+\mathrm{ve}$ \\
\hline 9. & ETP Inlet & Off White & Round & Elevated & Slimy & Cocci in chains & G-ve \\
\hline 10. & ETP Outlet & Off White & Lawn & Flat & Slimy & Cocci & G-ve \\
\hline
\end{tabular}

Table.3 Macroscopic and Microscopic visual characters of water samples from different sugar production stages of Uttar Pradesh Sugar Factory -II

\begin{tabular}{|l|l|l|l|l|l|l|l|}
\hline S.No & Sample & \multicolumn{2}{c|}{ Macroscopic Characters } & \multicolumn{2}{c|}{ Microscopic } \\
\cline { 3 - 6 } & & Colour & Shape & Elevation & Appearance & Shape & Gram stain \\
\hline 1. & Fresh water & Off White & Round & Flat & Slimy & Rods in chains & G-ve \\
\hline 2. & $1^{\text {st }}$ body condensate & Negligible Growth & & & & \\
\hline 3. & $2^{\text {nd }}$ body condensate & Negligible Growth & & & \\
\hline 4. & $3^{\text {rd }}$ body condensate & Negligible Growth \\
\hline 5. & $4^{\text {th }}$ body condensate & Negligible Growth \\
\hline 6. & Pan Condensate & & & & & \\
\hline 7. & Hot UGR & Off White & Lawn & Flat & Rough & Rods in chains & G-ve \\
\hline 8. & Cold UGR & Off White & Lawn & Flat & Smooth & Rods in chains & G-ve/ G+ve \\
\hline 9. & ETP Inlet & Off White & Round & Flat & Slimy & Rods & G-ve \\
\hline 10. & ETP Outlet & Off White & Lawn & Flat & Smooth & Rods in chains & G-ve \\
\hline
\end{tabular}

Table.4 Macroscopic and Microscopic visual characters of water samples from different sugar production stages of Maharashtra Sugar Factory -I

\begin{tabular}{|c|c|c|c|c|c|c|c|}
\hline \multirow[t]{2}{*}{ S.No } & \multirow[t]{2}{*}{ Sample } & \multicolumn{4}{|c|}{ Macroscopic Characters } & \multicolumn{2}{|c|}{ Microscopic } \\
\hline & & Colour & Shape & Elevation & Appearance & Shape & Gram stain \\
\hline 1. & DAM raw water & Off White & Lawn & Flat & Slimy & Cocci & G-ve \\
\hline 2. & $2^{\text {nd }}$ body condensate & \multicolumn{6}{|c|}{ Negligible Growth } \\
\hline 3. & $3^{\text {rd }}$ body condensate & \multicolumn{6}{|c|}{ Negligible Growth } \\
\hline 4. & $4^{\text {th }}$ body condensate & \multicolumn{6}{|c|}{ Negligible Growth } \\
\hline 5. & $5^{\text {th }}$ body condensate & \multicolumn{6}{|c|}{ Negligible Growth } \\
\hline 6. & Pan condensate & Off White & Lawn & Flat & Slimy & Cocci & G-ve \\
\hline 7. & Hot UGR & Off White & Lawn & Flat & Slimy & Cocci & G-ve \\
\hline 8. & Cold UGR & Off White & Lawn & Flat & Slimy & Cocci & G-ve \\
\hline 9. & ETP Inlet & Off White & Lawn & Flat & Rough & Rods & G-ve \\
\hline 10. & ETP Outlet & Off White & Lawn & Flat & Rough & Cocci & G-ve \\
\hline
\end{tabular}


Table.5 Macroscopic and Microscopic visual characters of water samples from different sugar production stages of Maharashtra Sugar Factory -II

\begin{tabular}{|c|c|c|c|c|c|c|c|}
\hline \multirow[t]{2}{*}{ S.No } & \multirow[t]{2}{*}{ Sample } & \multicolumn{4}{|c|}{ Macroscopic Characters } & \multicolumn{2}{|c|}{ Microscopic } \\
\hline & & Colour & Shape & Elevation & Appearance & Shape & Gram stain \\
\hline 1. & Raw water & Off White & Round & Flat & Rough & rods & G-ve \\
\hline 2. & $1^{\text {st }}$ body condensate & \multicolumn{6}{|c|}{ Negligible Growth } \\
\hline 3. & $2^{\text {nd }}$ body condensate & \multicolumn{6}{|c|}{ Negligible Growth } \\
\hline 4. & $3^{\text {rd }}$ body condensate & \multicolumn{6}{|c|}{ Negligible Growth } \\
\hline 5. & $4^{\text {th }}$ body condensate & \multicolumn{6}{|c|}{ Negligible Growth } \\
\hline 6. & Pan condensate & Off White & Lawn & Flat & Slimy & Cocci & G-ve \\
\hline 7. & Hot UGR & Off White & Lawn & Flat & Slimy & Rods & G-ve \\
\hline 8. & Cold UGR & Off White & Round & Flat & Rough & Rods & G-ve/ G+ve \\
\hline 9. & ETP Inlet & Off White & Round & Flat & Slimy & rods & G+ve \\
\hline 10. & ETP Outlet & Off White & Round & Flat & Rough & Rods & G+ve \\
\hline
\end{tabular}

Table.6 Macroscopic and Microscopic visual characters of water samples from different sugar production stages of Maharashtra Sugar Factory -III

\begin{tabular}{|c|c|c|c|c|c|c|c|}
\hline \multirow[t]{2}{*}{ S.No } & \multirow[t]{2}{*}{ Sample } & \multicolumn{4}{|c|}{ Macroscopic Characters } & \multicolumn{2}{|c|}{ Microscopic } \\
\hline & & Colour & Shape & Elevation & Appearance & Shape & Gram stain \\
\hline 1. & Raw water & Off White & Round & Flat & Slimy & Cocci / Rods & G-ve/ G+ve \\
\hline 2. & $1^{\text {st }}$ body condensate & \multicolumn{6}{|c|}{ Negligible Growth } \\
\hline 3. & $2^{\text {nd }}$ body condensate & \multicolumn{6}{|c|}{ Negligible Growth } \\
\hline 4. & $3^{\text {rd }}$ body condensate & \multicolumn{6}{|c|}{ Negligible Growth } \\
\hline 5. & $4^{\text {th }}$ body condensate & \multicolumn{6}{|c|}{ Negligible Growth } \\
\hline 6. & Pan Condensate & Off White & Round & Flat & Slimy & Rods & G+ve \\
\hline 7. & Hot UGR & & & & & & \\
\hline 8. & Cold UGR & & & & & & \\
\hline 9. & ETP Inlet & Off White & Lawn & Elevated & Slimy & Rods & G-ve \\
\hline 10. & ETP Outlet & Off White & Lawn & Elevated & Slimy & Rods & $\mathrm{G}+\mathrm{ve}$ \\
\hline
\end{tabular}

Table.7 Macroscopic and Microscopic visual characters of water samples from different sugar production stages of Tamil Nadu Sugar Factory -I

\begin{tabular}{|c|c|c|c|c|c|c|c|}
\hline \multirow[t]{2}{*}{ S.No } & \multirow[t]{2}{*}{ Sample } & \multicolumn{4}{|c|}{ Macroscopic Characters } & \multicolumn{2}{|c|}{ Microscopic } \\
\hline & & Colour & Shape & Elevation & Appearance & Shape & Gram stain \\
\hline 1. & Raw water & Off white & Lawn & Flat & Slimy & Cocci & G-ve \\
\hline 2. & $2^{\text {nd }}$ body condensate & \multicolumn{6}{|c|}{ Negligible Growth } \\
\hline 3. & $3^{\text {rd }}$ body condensate & \multicolumn{6}{|c|}{ Negligible Growth } \\
\hline 4. & $4^{\text {th }}$ body condensate & \multicolumn{6}{|c|}{ Negligible Growth } \\
\hline 5. & $5^{\text {th }}$ body condensate & \multicolumn{6}{|c|}{ Negligible Growth } \\
\hline 6. & Pan condensate & Off white & Lawn & Flat & Slimy & Cocci & G-ve \\
\hline 7. & Hot UGR & Off white & Lawn & Flat & Slimy & Rods & G+ve \\
\hline 8. & Cold UGR & Off white & Lawn & Flat & Slimy & Rods & G+ve \\
\hline 9. & ETP Inlet & Off white & Lawn & Flat & Rough & Cocci & G+ve \\
\hline 10. & ETP Outlet & Off white & Lawn & Flat & Rough & Cocci & G+ve \\
\hline
\end{tabular}


Table.8 Macroscopic and Microscopic visual characters of water samples from different sugar production stages of Tamil Nadu Sugar Factory -II

\begin{tabular}{|c|c|c|c|c|c|c|c|}
\hline \multirow[b]{2}{*}{ S.No } & \multirow[b]{2}{*}{ Sample } & \multicolumn{4}{|c|}{ Macroscopic Characters } & \multicolumn{2}{|c|}{ Microscopic } \\
\hline & & Colour & Shape & Elevation & Appearance & Shape & Gram stain \\
\hline 1. & Fresh water & Off white & Round & Flat & Slimy & Rods & G-ve \\
\hline 2. & $1^{\text {st }}$ body condensate & \multicolumn{6}{|c|}{ Negligible Growth } \\
\hline 3. & $2^{\text {nd }}$ body condensate & \multicolumn{6}{|c|}{ Negligible Growth } \\
\hline 4. & $3^{\text {rd }}$ body condensate & \multicolumn{6}{|c|}{ Negligible Growth } \\
\hline 5. & $4^{\text {rd }}$ body condensate & \multicolumn{6}{|c|}{ Negligible Growth } \\
\hline 6. & Pan Condensate & & & & & & \\
\hline 7. & Hot UGR & Off white & Lawn & Flat & Rough & Cocci & G+ve \\
\hline 8. & Cold UGR & Off white & Lawn & Flat & Smooth & Rods & G+ve \\
\hline 9. & ETP Inlet & Off white & Round & Flat & Slimy & Rods & G-ve \\
\hline 10 & ETP Outlet & Off white & Lawn & Flat & Smooth & Rods in chains & G-ve \\
\hline
\end{tabular}

Table.9 Biochemical evaluation of bacterial population and identification of bacterial strains from different sugar production stages of Uttar Pradesh Sugar Factory -I

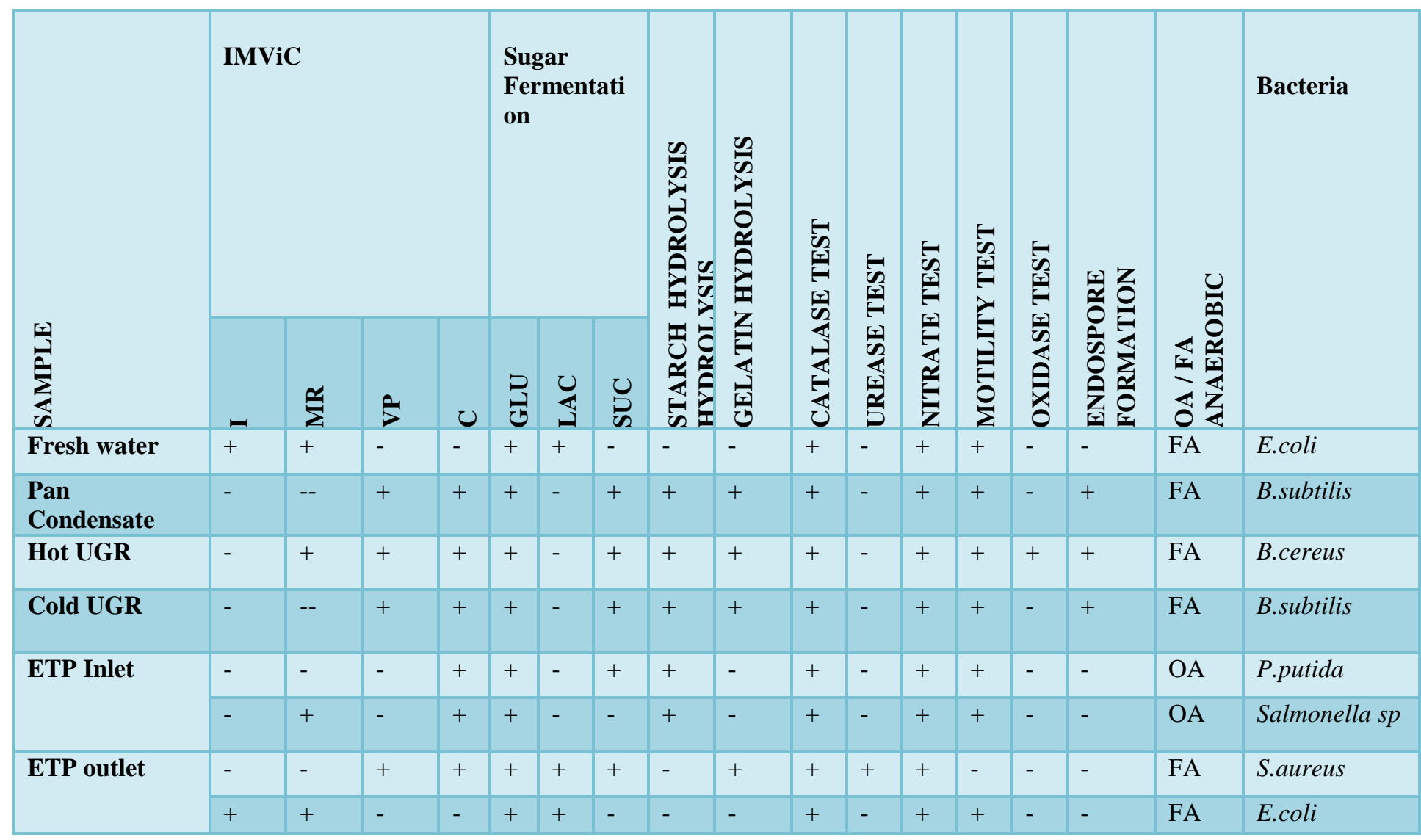


Table.10 Biochemical evaluation of bacterial population and identification of bacterial strains from different sugar production stages of Uttar Pradesh Sugar Factory -II

\begin{tabular}{|c|c|c|c|c|c|c|c|c|c|c|c|c|c|c|c|c|c|}
\hline \multirow{2}{*}{ 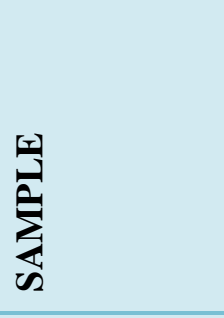 } & \multicolumn{4}{|c|}{ IMViC } & \multicolumn{3}{|c|}{$\begin{array}{l}\text { Sugar } \\
\text { Fermentati } \\
\text { on }\end{array}$} & \multirow{2}{*}{ 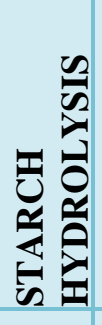 } & \multirow{2}{*}{ 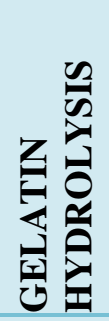 } & \multirow{2}{*}{ 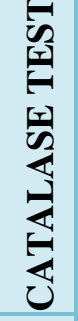 } & \multirow{2}{*}{ 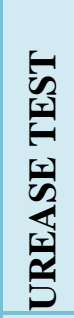 } & \multirow{2}{*}{ 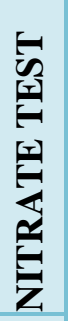 } & \multirow{2}{*}{ 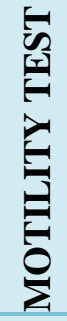 } & \multirow{2}{*}{ 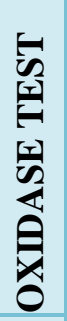 } & \multirow{2}{*}{ 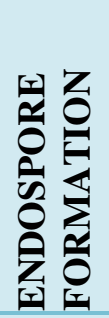 } & \multirow{2}{*}{ 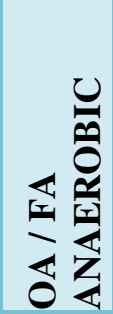 } & \multirow{2}{*}{ Bacteria } \\
\hline & - & $\stackrel{u}{\Sigma}$ & $\hat{z}$ & $U$ & ? & U & 己 & & & & & & & & & & \\
\hline \multirow[t]{2}{*}{ Fresh water } & + & + & - & - & + & + & - & - & - & + & - & + & + & - & - & FA & E.coli \\
\hline & - & + & - & + & + & - & - & + & - & + & - & + & + & - & - & OA & $\begin{array}{l}\text { Salmonella } \\
\text { spp. }\end{array}$ \\
\hline $\begin{array}{l}\text { Pan } \\
\text { Condensate }\end{array}$ & + & + & - & - & + & + & - & - & - & + & - & + & + & - & - & FA & E.coli \\
\hline $\begin{array}{l}\text { Hot UGR } \\
\text { inlet }\end{array}$ & - & + & + & + & + & - & + & + & + & + & - & + & + & + & + & FA & B.cereus \\
\hline $\begin{array}{l}\text { Cold UGR } \\
\text { inlet }\end{array}$ & + & + & - & - & + & + & - & - & - & + & - & + & + & - & - & FA & E.coli \\
\hline \multirow[t]{2}{*}{ ETP Inlet } & - & - & - & + & - & - & - & + & + & + & + & + & - & + & + & $\mathrm{OA}$ & $\begin{array}{l}\text { Micrococcus } \\
s p\end{array}$ \\
\hline & - & - & + & + & + & + & + & - & + & + & + & + & - & - & - & FA & S.aureus \\
\hline \multirow[t]{2}{*}{ ETP outlet } & - & - & - & + & - & - & - & + & + & + & + & + & - & + & + & $\mathrm{OA}$ & $\begin{array}{l}\text { Micrococcus } \\
s p\end{array}$ \\
\hline & - & - & + & + & + & + & + & - & + & + & + & + & - & - & - & FA & S.aureus \\
\hline
\end{tabular}

Table.11 Biochemical evaluation of bacterial population and identification of bacterial strains from different sugar production stages of Uttar Pradesh Sugar Factory -III

\begin{tabular}{|c|c|c|c|c|c|c|c|c|c|c|c|c|c|c|c|c|c|}
\hline \multirow{3}{*}{ 至 } & \multirow{2}{*}{\multicolumn{4}{|c|}{ IMViC }} & \multirow{2}{*}{\multicolumn{3}{|c|}{$\begin{array}{l}\text { Sugar } \\
\text { Fermentati } \\
\text { on }\end{array}$}} & \multirow{3}{*}{ 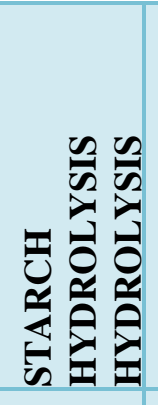 } & \multirow{3}{*}{ 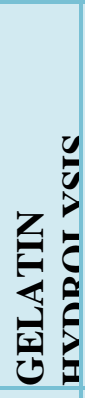 } & \multirow{3}{*}{ 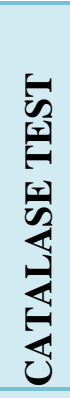 } & \multirow{3}{*}{ 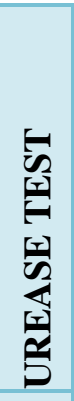 } & \multirow{3}{*}{ 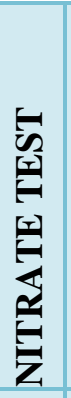 } & \multirow{3}{*}{ 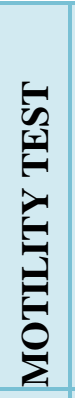 } & \multirow{3}{*}{ 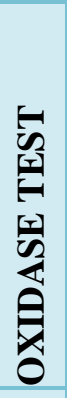 } & \multirow{3}{*}{ 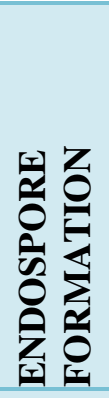 } & \multirow{3}{*}{$\sum_{0}^{\mathbb{1}}$} & \multirow{3}{*}{ Bacteria } \\
\hline & & & & & & & & & & & & & & & & & \\
\hline & - & $\stackrel{\underline{\Sigma}}{\Sigma}$ & $\hat{\nabla}$ & $u$ & 皇 & $\stackrel{3}{3}$ & U & & & & & & & & & & \\
\hline Fresh water & - & + & - & + & + & - & - & + & - & + & - & + & + & - & - & $\mathrm{OA}$ & $\begin{array}{l}\text { Salmonella } \\
s p\end{array}$ \\
\hline Hot UGR & - & + & - & + & + & - & - & + & - & + & - & + & + & - & - & $\mathrm{OA}$ & $\begin{array}{l}\text { Salmonella } \\
s p\end{array}$ \\
\hline \multirow[t]{2}{*}{ Cold UGR } & - & + & + & + & + & - & + & + & + & + & - & + & + & + & + & FA & B.cereus \\
\hline & + & + & - & - & + & + & - & - & - & + & - & + & + & - & - & FA & E.coli \\
\hline \multirow[t]{2}{*}{ ETP Inlet } & - & - & - & + & + & + & + & + & - & + & + & + & - & - & - & FA & Klebsiella sp. \\
\hline & + & + & - & - & + & + & - & - & - & + & - & + & + & - & - & FA & E.coli \\
\hline ETP Outlet & - & + & + & + & + & - & + & + & + & + & - & + & + & + & + & FA & B.cereus \\
\hline
\end{tabular}


Table.12 Biochemical evaluation of bacterial population and identification of bacterial strains from different sugar production stages of Maharashtra Sugar Factory -I

\begin{tabular}{|c|c|c|c|c|c|c|c|c|c|c|c|c|c|c|c|c|}
\hline \multirow{4}{*}{ 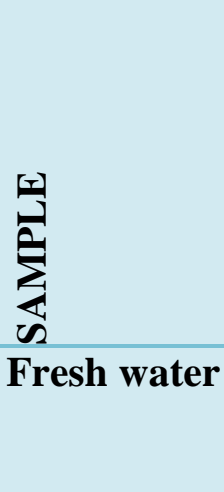 } & \multicolumn{4}{|c|}{ IMViC } & \multicolumn{3}{|c|}{$\begin{array}{l}\text { Sugar } \\
\text { Fermentati } \\
\text { on }\end{array}$} & \multirow{2}{*}{ 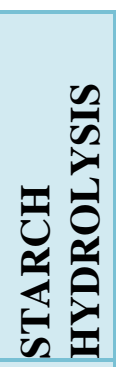 } & \multirow{2}{*}{ 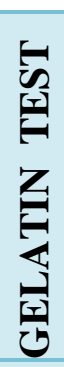 } & \multirow{2}{*}{ 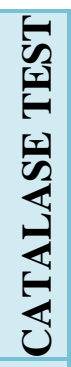 } & \multirow{2}{*}{ 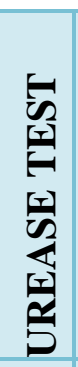 } & \multirow{2}{*}{ 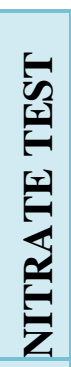 } & \multirow{2}{*}{ 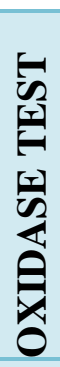 } & \multirow{2}{*}{ 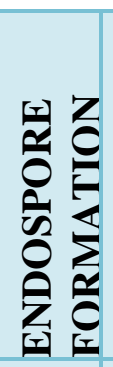 } & \multirow{2}{*}{$\underset{0}{\mathbb{Z}}$} & \multirow[t]{2}{*}{ Bacteria } \\
\hline & - & $\underline{\varepsilon}$ & $\hat{z}$ & $\circlearrowright$ & $\underset{7}{9}$ & $\underset{\ddots}{U}$ & $\stackrel{\vartheta}{\varrho}$ & & & & & & & & & \\
\hline & - & + & - & + & + & - & + & + & + & + & + & - & - & + & $\mathrm{OA}$ & $\begin{array}{l}\text { B.megateriu } \\
m\end{array}$ \\
\hline & - & -- & + & + & + & - & + & + & + & + & - & + & - & + & FA & B.subtilis \\
\hline $\begin{array}{l}\text { Pan } \\
\text { condensate }\end{array}$ & - & -- & + & + & + & - & + & + & + & + & - & + & - & + & FA & B.subtilis \\
\hline Hot UGR & + & + & - & - & + & + & - & - & - & + & - & + & - & - & $\mathrm{FA}$ & E.coli \\
\hline Cold UGR & + & + & - & - & + & + & - & - & - & + & - & + & - & - & FA & E.coli \\
\hline ETP Inlet & - & - & - & + & - & - & - & - & + & + & + & + & + & - & $\mathrm{OA}$ & $\begin{array}{l}P . \\
\text { aeruginosa }\end{array}$ \\
\hline & + & + & - & - & + & + & - & - & - & + & - & + & - & - & $\mathrm{OA}$ & E.coli \\
\hline ETP Outlet & - & + & - & + & + & - & - & + & - & + & - & + & - & - & $\mathrm{OA}$ & $\begin{array}{l}\text { Salmonella } \\
\text { sp }\end{array}$ \\
\hline & + & + & - & - & + & + & - & - & - & + & - & + & - & - & FA & E.coli \\
\hline
\end{tabular}

Table.13 Biochemical evaluation of bacterial population and identification of bacterial strains from different sugar production stages of Maharashtra Sugar Factory -II

\begin{tabular}{|c|c|c|c|c|c|c|c|c|c|c|c|c|c|c|c|c|c|}
\hline \multirow{2}{*}{$\sum_{\sqrt[S]{2}}^{\frac{5}{3}}$} & \multicolumn{4}{|c|}{ IMViC } & \multicolumn{3}{|c|}{$\begin{array}{l}\text { Sugar } \\
\text { Fermentat } \\
\text { ion }\end{array}$} & \multirow{2}{*}{ 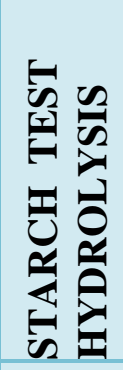 } & \multirow{2}{*}{$\frac{5}{5}$} & \multirow{2}{*}{ 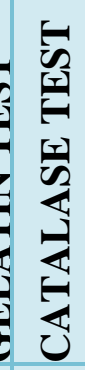 } & \multirow{2}{*}{ 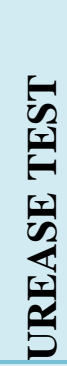 } & \multirow{2}{*}{ 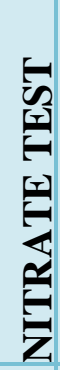 } & \multirow{2}{*}{ 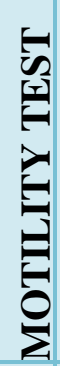 } & \multirow{2}{*}{ 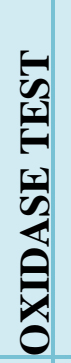 } & \multirow{2}{*}{$\begin{array}{l}\frac{1}{2} \\
0 \\
0 \\
0 \\
0 \\
0 \\
0\end{array}$} & \multirow{2}{*}{$\underset{0}{\pi}$} & \multirow[t]{2}{*}{ Bacteria } \\
\hline & - & $\underline{\Sigma}$ & a & $\circlearrowright$ & $\underset{\Xi}{\Xi}$ & $\underset{\Xi}{U}$ & $\stackrel{己}{\mathscr{D}}$ & & & & & & & & & & \\
\hline Raw water & + & + & - & - & + & + & - & - & - & + & - & + & + & - & - & FA & E.coli \\
\hline Hot UGR & - & - & - & + & - & - & - & - & + & + & + & + & + & + & - & $\mathrm{OA}$ & P. aeruginosa \\
\hline \multirow[t]{2}{*}{ Cold UGR } & + & + & - & - & + & + & - & - & - & + & - & + & + & - & - & FA & E.coli \\
\hline & - & -- & + & + & + & - & + & + & + & + & - & + & + & - & + & FA & B.subtilis \\
\hline \multirow[t]{2}{*}{ ETP Inlet } & - & + & + & + & + & - & + & + & + & + & - & + & + & + & + & FA & B.cereus \\
\hline & - & -- & + & + & + & - & + & + & + & + & - & + & + & - & + & FA & B.subtilis \\
\hline ETP Outlet & - & -- & + & + & + & - & + & + & + & + & - & + & + & - & + & FA & B.subtilis \\
\hline
\end{tabular}


Table.14 Biochemical evaluation of bacterial population and identification of bacterial strains from different sugar production stages of Maharashtra Sugar Factory -III

\begin{tabular}{|c|c|c|c|c|c|c|c|c|c|c|c|c|c|c|c|c|c|}
\hline \multirow{2}{*}{ 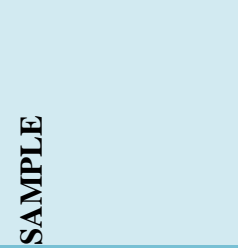 } & \multicolumn{4}{|c|}{ IMViC } & \multicolumn{3}{|c|}{$\begin{array}{l}\text { Sugar } \\
\text { Fermentation }\end{array}$} & \multirow{2}{*}{ 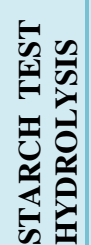 } & \multirow{2}{*}{ 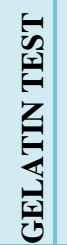 } & \multirow{2}{*}{ 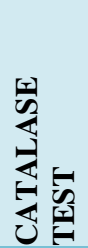 } & \multirow{2}{*}{ 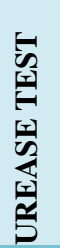 } & \multirow{2}{*}{ 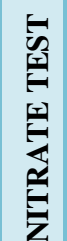 } & \multirow{2}{*}{ 帘 } & \multirow{2}{*}{ 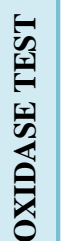 } & \multirow{2}{*}{ 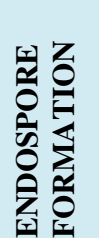 } & \multirow{2}{*}{$\underset{⿱ 乛}{\mathbb{S}}$} & \multirow[t]{2}{*}{ Bacteria } \\
\hline & $=$ & $\stackrel{\text { s }}{\Sigma}$ & $\overline{>}$ & U & 반 & $\Xi$ & U & & & & & & & & & & \\
\hline \multirow[t]{2}{*}{ Raw water } & - & - & + & + & + & + & + & - & + & + & + & + & - & - & - & FA & S.aureus \\
\hline & + & + & - & - & + & + & - & - & - & + & - & + & + & - & - & FA & E.coli \\
\hline Pan Condensate & - & -- & + & + & + & - & + & + & + & + & - & + & + & - & + & FA & B.subtilis \\
\hline \multirow[t]{2}{*}{ ETP Inlet } & - & - & - & + & - & - & - & - & + & + & + & + & + & + & - & $\mathrm{OA}$ & P. aeruginosa \\
\hline & + & + & - & - & + & - & + & - & + & + & + & + & + & - & - & FA & P.putida \\
\hline ETP Outlet & - & -- & + & + & + & - & + & + & + & + & - & + & + & - & + & FA & B.subtilis \\
\hline
\end{tabular}

Table.15 Biochemical evaluation of bacterial population and identification of bacterial strains from different sugar production stages of Tamil Nadu Sugar Factory -I

\begin{tabular}{|c|c|c|c|c|c|c|c|c|c|c|c|c|c|c|c|c|c|}
\hline \multirow{2}{*}{$\sum_{\text {is }}^{\sum_{1}}$} & \multicolumn{4}{|c|}{ IMViC } & \multicolumn{3}{|c|}{$\begin{array}{l}\text { Sugar } \\
\text { Fermentation }\end{array}$} & \multirow{2}{*}{\multicolumn{2}{|c|}{ 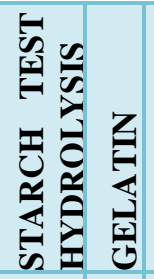 }} & \multirow{3}{*}{ 窗 } & \multirow{3}{*}{ 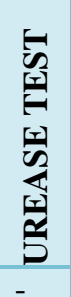 } & \multirow{3}{*}{ 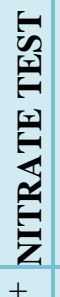 } & \multirow{3}{*}{ 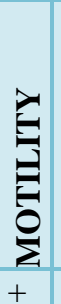 } & \multirow{3}{*}{ 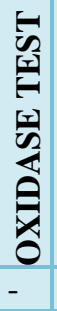 } & \multirow{2}{*}{\multicolumn{2}{|c|}{ 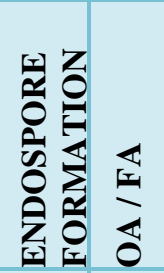 }} & \multirow[t]{2}{*}{ Bacteria } \\
\hline & - & $\underline{\Sigma}$ & $\hat{s}$ & u & $\overrightarrow{0}$ & 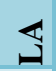 & 吊 & & & & & & & & & & \\
\hline Fresh water & - & + & - & + & + & + & + & - & - & & & & & & - & FA & Citrobacter spp. \\
\hline Hot UGR & - & - & + & + & + & + & + & - & + & + & + & + & - & - & - & FA & S.aureus \\
\hline Cold UGR & - & + & + & + & + & - & + & + & + & + & - & + & + & + & + & FA & B.cereus \\
\hline \multirow[t]{2}{*}{ ETP Inlet } & + & + & - & - & + & - & + & - & + & + & + & + & + & - & - & FA & P.vulgaris \\
\hline & - & + & - & + & + & + & + & - & - & + & - & + & + & - & - & FA & Citrobacter spp. \\
\hline ETP Outlet & + & + & - & - & + & + & - & - & - & + & - & + & + & - & - & FA & E.coli \\
\hline
\end{tabular}

Table.16 Biochemical evaluation of bacterial population and identification of bacterial strains from different sugar production stages of Tamil Nadu Sugar Factory -II

\begin{tabular}{|c|c|c|c|c|c|c|c|c|c|c|c|c|c|c|c|c|c|}
\hline \multirow{2}{*}{$\sum_{\text {至 }}$} & \multicolumn{4}{|c|}{ IMViC } & \multicolumn{3}{|c|}{$\begin{array}{l}\text { Sugar } \\
\text { Fermentation }\end{array}$} & \multirow{2}{*}{ 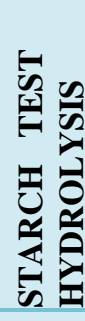 } & \multirow{2}{*}{ 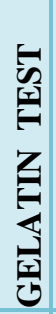 } & \multirow{2}{*}{ 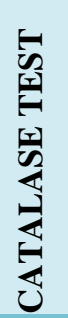 } & \multirow{2}{*}{ 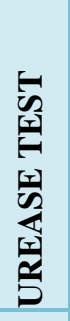 } & \multirow{2}{*}{ 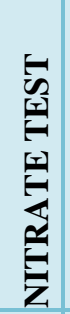 } & \multirow{2}{*}{ 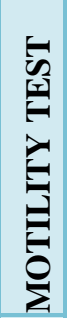 } & \multirow{2}{*}{ 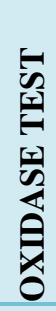 } & \multirow{2}{*}{ 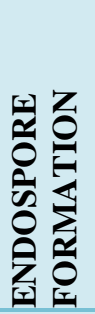 } & \multirow{2}{*}{$\frac{\overleftarrow{1}}{0}$} & \multirow{2}{*}{ Bacteria } \\
\hline & 二 & $\stackrel{\varrho}{\Sigma}$ & $\hat{z}$ & $u$ & 론 & $\underset{J}{U}$ & U & & & & & & & & & & \\
\hline Raw water & + & + & - & - & + & + & - & - & - & + & - & + & + & - & - & FA & E.coli \\
\hline Pan condensate & + & + & - & - & + & + & - & - & - & + & - & + & + & - & - & FA & E.coli \\
\hline Hot UGR & - & -- & + & + & + & - & + & + & + & + & - & + & + & - & + & $\mathrm{OA}$ & B.subtilis \\
\hline Cold UGR & - & + & + & + & + & - & + & + & + & + & - & + & + & + & + & FA & B.cereus \\
\hline ETP Inlet & - & - & + & - & + & + & + & - & + & + & + & + & - & - & - & FA & Leuconostoc \\
\hline ETP Outlet & - & - & - & + & + & + & + & - & - & + & + & - & - & - & - & $\begin{array}{l}\mathrm{OA} \\
\text { /FA }\end{array}$ & S.saprophyticus \\
\hline
\end{tabular}


Table.17 Macroscopic and Microscopic characters of fungal strains

\begin{tabular}{|c|c|c|c|}
\hline S.No & Macroscopic Characters & Microscopic Characters & Fungi \\
\hline \multicolumn{4}{|c|}{ Fresh Water } \\
\hline 1 & $\begin{array}{l}\text {-Pale white color, Texture deeply } \\
\text { cottony; } \\
\text {-White becoming gray-brown on } \\
\text { surface, } \\
\text {-Very rapid growth. }\end{array}$ & $\begin{array}{l}\text {-Hyphae broad, not or scarcely } \\
\text { septate; } \\
\text { - Rhizoids and stolons present; } \\
\text { - Sporangiophores brown, } \\
\text { sporangiophores } \\
\text { ovoid, sporangia rather round,. }\end{array}$ & Rhizopus \\
\hline \multicolumn{4}{|c|}{ ETP Inlet and Outlet } \\
\hline 2 & $\begin{array}{l}\text { Colonies are green, black or grey } \\
\text { in color. }\end{array}$ & $\begin{array}{l}\text {-Conidia are club shaped, } \\
\text {-Spores are single or form long } \\
\text { chains. }\end{array}$ & $\begin{array}{l}\text { Alternaria } \\
\text { gaisen }\end{array}$ \\
\hline 3 & $\begin{array}{l}\text {-Green in color with yellow or } \\
\text { white color margin. } \\
\text {-Wrinkled growth. } \\
\text {-Become black in color after } \\
\text { ageing. }\end{array}$ & $\begin{array}{l}\text {-Dense mats of mycelium with } \\
\text { conidia. } \\
\text {-Conidial heads are radiate. } \\
\text {-Conidia are globose to } \\
\text { subglobose. } \\
\text {-Pale green in color. }\end{array}$ & $\begin{array}{l}\text { Aspergillus } \\
\text { flavus }\end{array}$ \\
\hline 4 & $\begin{array}{l}\text {-White cottony growth. } \\
\text {-Sometime it gives pale yellow } \\
\text { color, black and shades of green. } \\
\text {-Erect conidiophores. }\end{array}$ & $\begin{array}{l}\text {-Conidia are one celled, smooth or } \\
\text { rough walled. } \\
\text {-Conidiophores consists of whorl } \\
\text { of phialids. }\end{array}$ & $\begin{array}{l}\text { Aspergillus } \\
\text { candidus }\end{array}$ \\
\hline 5 & $\begin{array}{l}\text {-Black in color. } \\
\text {-Powdery texture. } \\
\text {-Elevated growth. }\end{array}$ & $\begin{array}{l}\text {-Large dark brown conidial heads. } \\
\text {-Conidiophores are smooth-walled } \\
\text { and hyaline. } \\
\text {-Conidia are globose to } \\
\text { subglobose, dark brown to black } \\
\text { and rough walled. }\end{array}$ & $\begin{array}{l}\text { Aspergillus } \\
\text { niger }\end{array}$ \\
\hline 6 & $\begin{array}{l}\text {-Produced white, yellow, green } \\
\text { and brown color colonies. } \\
\text {-Cottony in texture. } \\
\text {-Slightly elevated. }\end{array}$ & $\begin{array}{l}\text {-Conidial heads are short } \\
\text { columnar. } \\
\text {-Conidiophores are usually short, } \\
\text { brownish and smooth walled. } \\
\text {-Conidia are globosend rough } \\
\text { walled. }\end{array}$ & $\begin{array}{l}\text { Aspergillus } \\
\text { nidulans }\end{array}$ \\
\hline 7 & $\begin{array}{l}\text {-Cottony growth. } \\
\text {-Center wrinkled. } \\
\text {-Mycelium white to orange white. } \\
\text {-Conidiogenesis moderate, grayish } \\
\text { green in color. }\end{array}$ & $\begin{array}{l}\text {-Colorless hyphae. } \\
\text {-Thallus highly branched. } \\
\text {-Constricted conidiophores. }\end{array}$ & $\begin{array}{l}\text { Peniciilium } \\
\text { pinophillum }\end{array}$ \\
\hline 8 & $\begin{array}{l}\text {-Light green in color and } \\
\text { yellowish conidia scattered } \\
\text { throughout the plate. }\end{array}$ & $\begin{array}{l}\text {-Conidia are globose. } \\
\text {-Phialids are slender. } \\
\text {-Branched conidiophores. }\end{array}$ & $\begin{array}{l}\text { Trichoderma } \\
\text { viride. }\end{array}$ \\
\hline
\end{tabular}


Fungal diversity is very important because of their economic importance as well as their Pathogenicity. Soon the basis of reported fungus and their ability of degradation and enzymatic activity of fungal strain they may use in commercial sector.

A large number of fungal diversity associated with sugarcane industrial effluent and this database created a novel record of the fungal diversity associated with sugarcane industrial effluent. Preparation of database provided a base in solving the problems associated with pollution of sugarcane industry and may become a basis for the management of sugarcane industrial effluent.

Due to the present study we can prepare a database which can provide a base in solving the problems associated with pollution of sugarcane industry and may become a basis for the management of sugarcane industrial effluent and recycle the less contaminated streams

The present investigation was carried out to isolate the most frequently occurring and optimally performing microorganisms from Sugar Industry. Wastewater exhibits dynamic characteristics, it is always better to use consortium over single culture Garcha $\mathrm{S}$ et al., (2014). Currently work is underway to construct the microbial consortia based on individual efficacy of isolates.

Mechanism of microorganisms in control of environmental pollution is still being explored. However, it is argued that organisms during bioremediation either eatup/ gobble the contaminants especially organic compounds or assimilate heavy metals themselves, thus effectively degrading specific contaminants / harmful compounds and converting them to nontoxic useable by products. Jai Shanker Pillai et al., (2011).

\section{Acknowledgements}

We would like to thank the Director NSI, Kanpur for providing all the facilities to carry out this research work.

\section{References}

Agarwal R, Sneh Lata, M Guptaeera, Singh P.2010, Removal of melanoidin present in distillery effluent as a major colorant: A Review, Journal of Environmental Biology, 31, pp 521-528.

Ashoka, C., Geetha, M.S., Sullia, S.B. 2002, Bioleaching of composite textile dye effluent using bacterial consortia. Asian, Journal of Microbial Biotechnology and Environmental Science, 4, pp 65-68.

Aust, S.D. 1990, Degradation of environmental pollutants, Microbial Ecology., 20, pp 197 - 209.

Avasan M., Rao S. R, 2009 Effect of sugar mill effluent on organic resources of fish, Pollution Research 20 (2 ) : 167171.

Awasthi A.K., Pandey.A.K., Rashmi Dubey 2011, Diversity of fungi in effluents of sugar industries of Madhya Pradesh, International Journal of Environmental Sciences.,1(5), pp 834-839.

Bergey's Manual. 1984, “Bergey's Manual of Systematic Bacteriology", Williams \& Wilkins, Baltimore: USA.

Bevan, 1971: The disposal of sugar mills effluents in Queensland 40th proceeding of the T.S.S.C.T. Louisinia October November PP $150 \mathrm{H}-1516$

Brown E., Skougstad M.W. Fishman M.J., 1970., Methods for collection and analysis of water samples for dissolved minerals and gases", Techniques of water resources investigations of the U.S. Geological Survey, 160.

Bumpus, J.A., Aust, S.D. 1993, Biodegradation of DDT (1, 1, 1trichloro $-2,2$ bis0) (4chlorophenyl) ethane) by 
the white - rot fungus phanerochaete chrysosporium", Appl. Environ. Microb, 53, pp 2001 - 2008.

Cappuccino, J.G., Sherman, N. 1999, "Microbiology; A Laboratory Manual", (34d edn.). Rockland Community College, Suffern: New York. Dahiya, J., D. Singh, P. Nigam (2001a), "Decolourisation of molasses wastewater by cells of Pseudomonas fluorescens immobilized on porous cellulose carrier”, Biores. Technol., 78, pp 111114.

CPCB 1989, corporate reasonability for Environmental protection (CREP) List of Industries under 'RED' Category Industries identified by Ministry of Environment \& Forests, Govt. of India as heavily polluting and covered under Central Action Plan.

Ellis, M.B (1976), "More Dematiaceous Hypomycetes", Commonwealth Mycological Institute Pub., Kew Surrey, England.

Garcha S., Brar S.K., Sharma K., Performance of a laboratory prepared microbial consortium for degradation of dairy waste water in a batch system, J. Sci. Ind. Res.73 (2014) 346-350.

Garcha S., Kaur N., Brar S.K., 2014, Aerobic granulation strategy for the treatment of dairy waste water, Indian J. Dairy Sci. $671-5$.

Gillman, J.C. 1957, A manual of soil fungi, Revised 2nd edn., Oxford and I.B.H Publishing company (Indian reprint), Calcutta, Bombay, New Delhi - 250.

Glazer, A.N.1997, Microbial Biotechnology, WH Freeman and Company. New York. pp. 54-58.

Jai Shanker Pillai Naveen Danesh, Puttaiah .E.T, Girish K. 2011, Microbial diversity in solid waste molasses of Sugar Industry, Aranthangi, Tamilnadu" International Journal of Environmental Sciences Volume 2, No 2.
Jimenez, A.M., Borja, R., Martin, A. 2003, Aerobicanaerobic biodegradation of beet molasses alcoholic fermentation wastewater, Process Biochemistry, 38, pp 1275-1284.

Josey,D.P.,Beyhon, J.L., Johnson, A.W.B., Beringer, J.E. 1979, "Strain identification in Rhizobium using intrinsic antibiotic resistance", Journal of Applied. Bacteriology., 46, pp 343350.

Miranda, P.M., Benito, G.G., Cristobal, N.S., Nieto, C.H. 1996, Colour elimination from molasses wastewater by Aspergillus niger, Bio-resource Technology, 57, pp 229-235.

Ohmomo, S. 1988, Screening of anaerobic bacteria with the ability to decolourize molasses melanoidin", Agricultural and biological chemistry, 57, pp 2429-2435.

Ohmomo, S. 1987, Decolourization of molasses wastewater by a thermophilic strain Aspergillus fumigatus G26, Agricultural and biological chemistry, 51, pp 3339-3346.

Pant, D., Adholeya, A.2007b, "Biological approaches for treatment of distillery wastewater: a review", Bioresource Technology, 98, pp 2321-2334.

Rahman, K.S.M., Banat, I.M., Rahman, T.J., Thayumanavan, T.,

Lakshmanaperumalsamy, P. 2002, "Bioremediation of gasoline contaminated soil by a bacterial consortium amended with poultry litter, coir pith and rhamnolipid biosurfactant", Bioresource Technology., 81, pp 2532.

Rajeshwarai K.V., Balakrishnan M., Kansal A., Kusumlata, Kishore V.V.N., 1999, State-of-the-art of anaerobic digestion technology for industrial wastewater treatment, Renewable and sustainable Energy Reviews 4 (2000) 135-156.

Ramlake, P.W., Bhattacharjee, J.W. 1992, Bacterial pollution of drinking water 
sources in north Tripura district, Proc Acad Environ Bio, 1(1), pp 1926.

Satyawali, Y., Balakrishnan, M. 2007, "Removal of color from biomethanated distillery spent wash by treatment with activated carbons", Bio-resource Technology, 98, pp 2629- 2635.

Somasegaran and Hoben. 1985, In : Methods in Legume - Rhizobium Technology, University of Hawaii, NIFTAL, Project and Micren, Dept. of Agronomy and Soil., pp 1300.

Street, R.A., Kulkarni, M.G., Strik, W.A., Southway, C., Van Staden, J. 2007, Toxicity of metal elements on germination and seedling growth of widely used medicinal plants belonging to Hyacinthaceae, Bulletin of Environmental Contamination and Toxicology, 79, pp 371-376.

Subramaniam, A., Brown, C.W., Hood, R.R., Carpentor, E.J., Capone, D.G. 2002, Detecting Trichodesmium blooms in Sea WiFS imagery, Deep Sea Res., 49, pp 107-121.

Suhuttaya Jiranuntipon. 2009, Decolorization of molasses wastewater from distilleries using bacterial consortium', Dissertation, DAMRONGLERD, Dr.Ing., pp 183209.

Vinish kathuria, 2014, Small Industry in India: Are CETPs an Appropriate Response for Controlling Pollution? (PP.245-260).

\section{How to cite this article:}

Seema Paroha and Shikha Singh. 2020. Investigations on Microbial Flora Variation in Water Generated at Various Sugar Production Stages. Int.J.Curr.Microbiol.App.Sci. 9(05): 10141028. doi: https://doi.org/10.20546/ijcmas.2020.905.111 